Indian plants must have been done since the manuscript of the book went to press fourteen years ago. The authors, in their desire for completeness, even go so far as to describe the symptoms and treatment of persons suffering from poisoning.

Although a great deal of information is given about a large number of species, it is surprising to note the number of plants which are "reported" or "said" to contain poisonous principles, and upon which no work has yet been done. This is a measure of the task which lies before Indian chemists and pharma. cognosists during the years to come.

The book is illustrated by a number of linedrawings of very unequal merit. Some of them are reasonably good, while others are quite unworthy of a book of this importance: some are from works fifty years old, while others have come from books published a hundred or even a hundred and fifty years or more ago. A serious defect in the illus. trations is that in no instance is there any indication of the size of the parts illustrated, or even what these parts may be, although a number of the blocks contain dissections. If it is thought necessary to have illustrations, then these should be good. The binding is quite inadequate for a book of this weight (4 lb.) and will definitely not stand up to the very frequent occasions on which the volume will be consulted.

The authors are to be congratulated on a really useful book, which for many years to come will be indispensable to all medical and botanical libraries as a work of reference. N. L. Bor

\section{TURBULENT MOTION OF FLUIDS}

The Theory of Homogeneous Turbulence

By Dr. G. K. Batchelor. (Cambridge Monographs on Mechanics and Applied Mathematics.) Pp. xi+197. (Cambridge: At the University Press, 1953.) 25s. net.

$7 \mathrm{O}$ obtain some understanding of the 'turbulent' motion of fluids (a term first used by Kelvin) has been one of the outstanding problems of the physical sciences since 1883, when Osborne Reynolds initiated the subject in a series of brilliant papers. In the succeeding age, when the problems most discussed by physicists were on the atomic and subatomic scale, the problem of turbulence remained a notable exception and continued to excite the imaginations of the most eminent students of the physical sciences. Werner Heisenberg, for example, has constantly returned to the subject from his better-known researches in quantum theory, and Rayleigh, Sommerfeld and Kolmogorov all made contributions. Ludwig Prandtl, the creator of the modern dynamies of fluids, who died recently, and his pupil von Kármán, have been important influences at all stages of the work. But perhaps the most single-minded, and successful, of the many warriors assailing the dragon has been G. I. Taylor who, though approaching his seventieth birthday, is still extremely active in the fight. After tackling the problem from many different and valuable points of view, he initiated in 1935 a new approach. Recognizing at last that all the methods based on the conventional ideas of statistical mechanics were doomed to failure, he developed a statistical theory of turbulence founded on the recognition that fluids behave like continua, with the statistical correlation coefficient between the fluid velocities at two nearby points as the fundamental observable. Later he demonstrated the relation of this correlation to the spatial spectrum of turbulence.

The new methods have been applied principally to the particular case of 'homogeneous' turbulence-that is to say, turbulence the statistical properties of which do not vary from point to point. The difficulties of extending them to non-homogeneous turbulence appear formidable; but the qualitative ideas suggested by the statistical theory, and windtunnel results, for homogeneous turbulence are already useful in all problems of turbulence. Since the Second World War, progress in homogeneous turbulence has been nowhere so great as in Taylor's own research group in the Cavendish Laboratory, Cambridge, led by $G$. K. Batchelor and A. A. Townsend. In the circumstances, no one could have been more suitable than Dr. Batchelor for giving a complete account of the present state of knowledge of the subject, and he has succeeded admirably in the book under review. Developing the subject in a logical order, he makes the reader thoroughly familiar with all the detailed correlations and spectra and their simpler, purely kinematical properties, before advancing to the problem of the general dynamies of decay, where one must make use of the fact that each realization of the velocity field satisfies the momentum equation of Stokes and Navier. The need for additional postulates like the 'local similarity' theory of Kolmogorov and the quasi-equilibrium theories of later authors is clearly shown, and these theories are critically explained. The probability distributions of the velocity and its derivatives are described, with physical inferences about the smallest-scale features of turbulence, and the use of the hypothesis that mean products of four velocities are related to mean products of two as for quantities obeying a joint normal distribution law, is illustrated by the calculation of the pressure correlation function. The bibliography is comprehensive, and the general layout of the book (the first of a new Cambridge series) makes for easy and pleasant reading. The dedication is "To G. I.".

If, less than two years after the book was com. pleted, the subject has moved on, this is due almost entirely to further work in the Cavendish Laboratory by Dr. Batchelor, Dr. Townsend and their associates. If the book were being re-edited, much less would be said about the general tendency to isotropy of homogeneous turbulence, which seems to be unsubstantiated for the eddies containing most of the turbulent energy. The theory that the joint distribution of velocities becomes normal in the final period of decay, so that different spectral components of the velocity are statistically independent, seems untenable because the different components each decay exponentially in that period, conserving whatever statistical dependence they may already have. Again, the theory of the 'permanence of big eddies' is currently being questioned. Dr. I. Proudman has calculated the sound field which isotropic turbulence generates.

All the time, in fact, the extent of our pitifully small understanding of turbulent motion is increasing. If all students of the subject make themselves thoroughly familiar with the material of Dr. Batchelor's book, so that they obtain a clear idea of the established knowledge without being encouraged to gloss over any real difficulties or unsimplifiable intricacies, then one may expect future progress to become faster still. M. J. LramTHILL 